\title{
Variations in Mean Platelet Volume in Patients with Helicobacter pylori Infection before and after Eradication, Way before Immune Thrombocytopenia? ${ }^{\dagger}$
}

\author{
Burcu Dag 1, Elif G. Umit 1,* and Hasan Umit ${ }^{2}$ \\ 1 Department of Hematology, Trakya University Faculty of Medicine, Edirne 22030, Turkey; \\ burcudag@trakya.edu.tr \\ 2 Department of Gastroenterology, Trakya University Faculty of Medicine, Edirne 22030, Turkey; \\ hasanumit@trakya.edu.tr \\ * Correspondence: elifgumit@trakya.edu.tr or egugur@yahoo.com; Tel.: +90-505-396-3996 \\ + Presented at the 25th Biennial International Congress on Thrombosis, Venice, Italy, 23-26 May 2018. \\ Published: 27 July 2018
}

\begin{abstract}
Helicobacter pylori (H. pylori) and immune thrombocytopenia (ITP) association is well known and eradication treatment has its place in both treatment guidelines. Since H. pylori eradication is followed by an increase in platelet counts in patients with immune thrombocytopenia, it is suggested that $H$. pylori be examined and treated if infection is present. There is only one study that demonstrated a relation between $H$. pylori and platelet indices in individuals with normal platelet counts. In this study, we aimed to investigate the effects of $H$. pylori infection on platelet count and mean platelet volume, which is a sign of increased platelet destruction in patients with normal platelet counts. We evaluated the data of 106 patients with urease test positivity before the eradication of bacteria and urea breath test negative after the eradication, in a retrospective manner. Mean platelet count in patients before the eradication treatment was $256.730 \pm 66.380 / \mathrm{mm}^{3}$. After $H$. pylori was eradicated, it has been observed that the mean platelet count increased to $287.080 \pm$ $59.240 / \mathrm{mm}^{3}$. Mean MPV of patients before and after eradication treatment were $9.35 \pm 1.63 \mathrm{fL}$ and $8.61 \pm 1.48 \mathrm{fL}$. Mean MPV was higher when patients were infected with $H$. pylori. This study showed that there is an increase in platelet counts and a decrease in MPV levels with the eradication of the H. pylori. Our study is the first to investigate changes of mean platelet volume and platelet count before and after eradication of $H$. pylori infection in individuals with normal platelet counts.
\end{abstract}

Keywords: Helicobacter pylori; immune thrombocytopenia; mean platelet volume

\section{Introduction}

H. pylori is a Gram-negative micro-aerophilic bacterium that colonizes the human gastric mucosa. H. pylori infection is one of the most common bacterial infections worldwide and its prevalence has been estimated to extend from 40 to $80 \%$ and it changes widely by geographic region, age, race, ethnicity, and socioeconomic factors. H. pylori strains are highly diverse and this diversity is associated with a variable host immune response that may contribute to the outcome. H. pylori is involved in the pathogenesis of chronic active gastritis as well as duodenal ulcer, gastric ulcer and gastric cancer. In addition to its well-demonstrated role in gastroduodenal diseases, $H$. pylori infection has been associated with a diverse spectrum of non-digestive system diseases including idiopathic thrombocytopenic purpura, iron deficiency anemia and some autoimmune conditions, such as rheumatoid arthritis and thyroid diseases.

Platelets have essential roles in thrombosis and hemostasis. The normal platelet count ranges from 100,000/L to 450,000/L. Circulating lifespan of platelets is 10 days. Maintain the adequate platelet 
count a regular and continuous balance between production and consumption is required. In thrombocytopenia which is defined as platelet count being less than $150 \times 109 / \mathrm{L}$, platelet size may be suggestive of a specific etiology. Large platelets suggest a process of platelet destruction leading to the production of younger and larger platelets. In thrombocytopenic patients, normal platelet volume may indicate an impaired thrombopoiesis.

Idiopathic thrombocytopenic purpura (ITP) is an acquired bleeding disorder in which autoantibodies bind to platelet surface, leading to platelet destruction. However, the factors that incite this disorder remain uncertain, though some infectious agents have been thought to be responsible for the formation of platelet antibodies.

Although $H$. pylori and ITP association is well documented and eradication treatment has its place in $H$. pylori and ITP treatment guidelines, there are not enough studies about the effects of $H$. pylori on platelet counts and volumes in patients who has normal platelet counts. In this study, we have aimed to examine the platelet count and the mean platelet volume changes following H. pylori eradication in patients who have normal platelet counts and no chronic diseases except for dyspepsia.

\section{Patients and Methods}

For this retrospective study, we studied patients who had no chronic diseases other than dyspepsia and were diagnosed with $H$. pylori infection by using endoscopic urease test between the years 2011-2016. Among these patients who given a succesful eradication treatment, as proven by urea breahth test, were included in this study. Since patients whose eradication therapy was not successful and data was accessible was few for a reliable assessment, they were excluded of this study. Patients who had malignant lesions, who had a gastrointestinal system bleeding in the past 3 months, who were subjected to blood and blood product transfusions in the past 3 months, who have systemic malignancy, who use drugs that might have an effect on their platelet counts or functions and who have thrombocytopenia were not included in our study. Between the years 2011-2016, when our study took place, blood counts were conducted with Sysmex and Beckman coulter LH 780 autoanaylzers at our faculty. EDTA tubes were used for the blood counts of the patients included in our study. Since this is a retrospective study, while it is not known exactly when the MPV measurements took place after the blood was drawn from the patients, but all the blood samples which are drawn at our hospital reach laboratory and blood count tests are concluded within $2 \mathrm{~h}$.

\section{Results}

The mean age of 106 patients who were included in this study was $50.14 \pm 13.31$ years old. The ages of the patients varied between 22 and 79 . While 61 patients $(57.5 \%)$ were female, 45 patients $(42.5 \%)$ were male. While the mean platelet count of the patients before eradication therapy was $256.730 \pm 66.380 / \mathrm{mm}^{3}$, it's been observed that mean platelet count became $287.080 \pm 59.240 / \mathrm{mm}^{3}$ after eradication of $H$. pylori. It has been observed that there was a significant increase $(p<0.001)$ in the platelet count following the eradication of H. pylori. When we examined the MPV values, it's been observed that the MPV values were $9.35 \pm 1.63 \mathrm{fL}$ before eradication, and they became $8.61 \pm 1.48 \mathrm{fL}$ after the eradication of H. pylori. A significant decrease $(p<0.001)$ in the MPV values following the eradication of $H$. pylori has been observed. For both sexes, there was an increase in the mean platelet count and a decrease in the mean platelet volume following the H. pylori eradication. There was no significant difference between sexes. When the patients were grouped according to their age as patients aged under 45 and over 45 , it has been observed that there was an increase in platelet count and a decrease in the mean platelet volume in both age groups following the eradication of the bacteria. Eradication showed positive effects on both age groups.

\section{Discussion}

In this study we evaluated patients for $H$. pylori infection by urease test. Urease test is a reliable method for the detection of $H$. pylori with a sensitivity of $80-100 \%$ and specificity of $97-99 \%$. The urea breath test was used for checking eradication of the bacteria. The urea breath test is a noninvasive 
test for diagnosis of gastric $H$. pylori infection. The test consists of drinking carbon-13 labeled or carbon-14 labeled urea. The ingested radiolabeled carbon is converted to carbon dioxide and ammonia by the urease created by the H. pylori. The carbon dioxide is absorbed in the bloodstream and a sample of expired air is analyzed for the presence of the labeled carbon. The sensitivity and specificity for the urea breath test is $99 \%$ and $98 \%$, respectively. Proton pump inhibitors, H2 receptor antagonists, bismuth and antibiotics whether given specifically as a treatment for $\mathrm{H}$. pylori or not need to be withdrawn for several days prior to testing due to the potential for false negatives. Breath testing should not be performed until at least one month after the end of such a course of treatment. In our laboratory we abide this rule. Because of this we think at least one month prior to urea breath test these patients did not take such treatments. Considering circulating lifespan of platelets is only 10 days, our examined platelet counts and MPV values after the urea breath test are results of the newly produced platelets. We tried to eliminate other factors that may affect platelet counts and MPV values with the exclusion criteria of study. The increase in the platelet count and the decrease in MPV values can be attributed to eradication of the $H$. pylori infection. Planning of a controlled prospective study about this subject will be very important in elimination of unevaluated factors.

Beneficial effect of $H$. pylori eradication on platelet counts in ITP patients has been reported [14]. Following that study several clinical studies have been demonstrated. Several hypotheses have been proposed regarding the mechanism by how $H$. pylori might induce thedevelopment of ITP. It has been proposed that $H$. pylori could induce antibody production in response to antigens that crossreact to various platelet glycoprotein antigens. In addition to low platelet counts increased MPV values too another important index that shows platelet destruction. Although H. pylori ITP association is well documented in certain group of ITP patients, the effect of $H$. pylori over MPV values has not been assessed enough [1]. MPV has been the subject of only 2 small studies and these studies have focused on the correlation of $H$. pylori infection intensity with MPV values and observed no correlation. However, they have not referred to the effect of $H$. pylori infection on platelet counts or MPV values.

There is only one study to investigate the effect of $H$. pylori in patients with normal platelet counts. This study compared the MPV values of $H$. pylori-infected and non-infected patients with normal platelet counts. Mean platelet count in $H$. pylori positive and negative patients were $24.6381 \pm$ $92.225 / \mathrm{mm}^{3}$ and $25.8135 \pm 89.912 / \mathrm{mm}^{3}$, respectively $(p<0.001)$. Mean MPV was higher in $H$. pylori positive group $(8.9 \pm 1.3$ vs. $8.23 \pm 0.94, p<0.001)$. This difference was observed in both genders. This is the first study to show that MPV values is increased in H. pylori positive individuals [5]. In our study instead of two separate groups of patients, we compared blood count of same patients before and after $H$. pylori eradication. While the patients were infected with $H$. pylori, the mean platelet counts were $256.730 \pm 66.380 / \mathrm{mm}^{3}$ and MPV values were $9.35 \pm 1.63 \mathrm{fL}$. It has been observed that the median platelet count increased to $287.080 \pm 59.240 / \mathrm{mm}^{3}$ and MPV values regressed to around $8.61 \pm$ $1.48 \mathrm{fL}$ after bacteria eradication. We observed no difference between sexes $(p<0.001)$. Our study is the first to show that there is an increase in absolute platelet counts and a decrease in MPV values with the eradication of the H. pylori in patients with normal platelet counts. Because our study is retrospective, there may be confounding factors that may effected the main findings. For preventing this to happen, we did a detailed patient record and tried to exclude confounding factors.

\section{Conclusions}

We can assume that for indivuals with a normal platelet count, chronic $H$. pylori infection causes a chronic compensated platelet destruction and production. This companseted destruction and production is effecting mean platelet volumes even though platelet counts never reaches the thrombocytopenic amounts. It can be considered that factors related to $H$. pylori strains or to the infected host can accelerate this process and cause thrombocytopenia.

Our study is the first study which shows that there might be an increase in the platelet count and a decrease in the mean platelet volumes after the eradication of $H$. pylori in patients with normal platelet counts. We hope that this study encourages wider-scaled, prospective and controlled further studies on this subject. 
Author Contributions: E.G.U. conceived and designed the study; B.D. performed the data collection; E.G.U. and H.U. analyzed the data; B.D. and E.G.U. wrote the paper.

Acknowledgments: This study was not funded by any authority or entity.

Conflicts of Interest: The authors declare no conflict of interest.

\section{References}

1. Michel, M.; Cooper, N.; Jean, C.; Frissora, C.; Bussel, J.B. Does Helicobater pylori initiate or perpetuate immune thrombocytopenic purpura? Blood 2004, 103, 890-896.

2. Topal, F.; Karaman, K.; Akbulut, S.; Dincer, N.; Dolek, Y.; Cosgun, Y.; Yonem, O. The relationship between mean platelet volume levels and the inflammation in Helicobacter pylori gastritis. J. Nat. Med. Assoc. 2010, $102,726$.

3. Yeniova, A.O.; Kucukazman, M.; Ata, N.; Dal, K.; Kefeli, A.; Bulus, H.; Akin, K.O.; Ertugrul, D.T.; Nazligul, Y. Investigation of the association between mean platelet volume and Helicobacter pylori gastritis. Afr. J. Microbiol. Res. 2013, 7, 2179-2183.

4. Beyan, C. Mean platelet volume for distinguishing between inherited thrombocytopenias and immune thrombocytopenia. Br. J. Haematol. 2013, 163, 412-413.

5. Umit, H.; Umit, E. Helicobacter pylori and mean platelet volume: A relation way before immune thrombocytopenia. Eur. Rev. Med. Pharmacol. Sci. 2015, 19, 2818-2823.

(C) 2018 by the authors. Licensee MDPI, Basel, Switzerland. This article is an open access article distributed under the terms and conditions of the Creative Commons Attribution (CC BY) license (http://creativecommons.org/licenses/by/4.0/). 